\title{
¿RACIONALIDAD DE LAS NECESIDADES EN EL DERECHO PRIVADO?
}

\section{Teorías sobre la racionalidad de las necesidades en el Derecho}

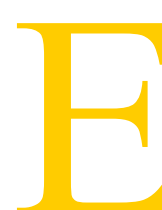

$\mathrm{n}$ la discusión teorético-histórica sobre las estructuras de la racionalidad en el Derecho, los teóricos presentan a menudo una noción de desarrollo evolutivo en diferentes etapas ${ }^{1}$. En muchos casos, el objetivo de estos análisis es mostrar que el Derecho está a punto de alcanzar una nueva etapa y que los indicios de esta nueva etapa pueden encontrarse ya en el sistema jurídico. Normalmente, estas teorías contienen, también, un elemento de normatividad: la nueva etapa es algo jurídico y los juristas teóricos deberían luchar por ello².

Una teoría de este tipo ha sido presentada en Finlandia por el teórico marxista Lars D. Eriksson ${ }^{3}$. Esta teoría, centrada principalmente en el desarrollo de los modelos de argumentación jurídica en la sociedad capitalista, distingue tres etapas:

a) La racionalidad del valor de cambio del capitalismo temprano forma la base del modelo de argumentación de la lógica de la subsunción. En este modelo, el punto de partida de la argumentación está constituido por la norma jurídica misma y la previsibilidad de las decisiones jurídicas es un valor central.

b) En el capitalismo tardío la racionalidad del valor de uso alcanza una posición dominante. Este tipo de racionalidad forma, sin embargo, la base de dos modelos diferentes de argumentación

\footnotetext{
${ }^{1}$ Para una crítica general de estas teorías evolutivas, véase, p. ej., Rottleuthner (1986): «El precio de la elegancia de un modelo de evolución es un desvanecimiento parcial».

${ }^{2}$ Naturalmente, hay también teóricos que advierten en contra de los nuevos rasgos que presenta el desarrollo jurídico. En Escandinavia hay que mencionar especialmente a Thomas Mathiesen (1985), quien, junto con muchos otros, diagnostica una evolución desde un método de regulación cerrado (basado sobre la certeza del Derecho) hacia un método de regulación más abierto; y, en su opinión, el declive de la certeza hará del Derecho un instrumento aún más efectivo para la opresión de los grupos más débiles de la sociedad.
}

${ }^{3}$ Véase Eriksson, 1979 y 1980, pág. 108. 
jurídica. El primero es el modelo de la racionalidad del fin (o modelo de racionalidad del fin orientado hacia el sistema). En este modelo el contenido social de las decisiones jurídicas pasa a ocupar un primer plano. Los fines de las regulaciones jurídicas se toman como punto de partida y se evalúan las consecuencias sociales de las decisiones. El que toma la decisión procura alcanzar unos resultados sociales equilibrados que contribuyen a la conservación del sistema.

c) La racionalidad del valor de uso está también detrás del modelo de argumentación de la racionalidad de las necesidades (o modelo de racionalidad del fin orientado hacia las necesidades). En este modelo se pone también el énfasis en el contenido social y en las consecuencias de la decisión jurídica. Sin embargo, el objetivo no es cuidar de las necesidades del sistema (de su cohesión), sino de las necesidades de los miembros de la sociedad. En la evaluación de la racionalidad de una decisión la cuestión crucial es qué efectos produce dicha decisión en relación con las necesidades concretas y reales de los miembros de la sociedad.

Esta última etapa en parte se presenta como una descripción de una nueva estructura de la racionalidad que ya puede observarse en algunos elementos del Derecho. Esta es, sin embargo, una teoría con un marcado contenido normativo. Un jurista «progresista», de acuerdo con esta teoría, tiene que luchar por consolidar los elementos de racionalidad de las necesidades presentes en el Derecho. La racionalidad de las necesidades se presenta, pues, como un concepto clave para una jurisprudencia «alternativa».

La teoría de la racionalidad de las necesidades parece tener algunas semejanzas interesantes con la muy debatida teoría del «Derecho reflexivo», de Gunther Teubner" ambas teorías -como muchas otras ${ }^{5}$ - dividen la evolución del Derecho en la sociedad capitalista en tres etapas, la última de la cuales se está empezando a desarrollar ahora. Esta última fase, en cierto sentido utópica, de las teorías de Teubner y Eriksson no se parece demasiado en uno y otro caso: mientras que la racionalidad de las necesidades presupone y acepta un contenido material del Derecho, el Derecho reflexivo no busca soluciones materiales,

${ }^{4}$ Véase, p. ej., Teubner, 1983.

${ }^{5}$ Véase, p. ej., Schmidt, 1980 (Privatautonomie - Materialisierung - Soziallautonomie), Brüggemeier, 1982 (contract I, contract II, organisation, y Wiethölter, 1982, 1984 (Formalisierung Materialisierung - Prozeduralisierung). 
sino que trata de garantizar las premisas estructurales para un proceso genuinamente reflexivo (auto-regulación). Las partes (grupos) implicadas tienen que tener ellas mismas la posibilidad de decidir el contenido normativo de sus relaciones. El Derecho solamente ha de «decidir sobre decisiones, regular regulaciones, y establecer las premisas estructurales para futuras decisiones en términos de organización, procedimiento y competencia» ${ }^{6}$. Sin embargo, una mirada atenta a la teoría del Derecho reflexivo revelará también algún contenido material, al menos en una perspectiva a corto plazo. Dentro de su doctrina, Teubner alude a la «lógica reflexiva», una lógica que implica «simular» un proceso reflexivo en los casos en que las asimetrías sociales de poder y de información pueden suponer un obstáculo para una genuina reflexividad. Esta lógica se implementa a través de cláusulas generales, recurre a estándares como la «buena fe» y el «interés público». Con la ayuda de estas cláusulas generales el Derecho puede crear normas «reflexivas» particulares con un contenido material: «Ello significa que, en el caso de "deficiencias en la interacción" entre las partes contratantes, se definan autorizadamente en virtud del Derecho fines y deberes objetivos; en el caso de "deficiencias en el mercado", la costumbre mercantil se sustituya por la definición judicial de las reglas de conducta del mercado; y en el caso de “deficiencias políticas" el proceso judicial defina estándares de interés público» ${ }^{7}$. En este proceso simulado -que parece ser un tipo de estrategia a corto plazo del Derecho reflexivoes obvio que debe ponerse algún énfasis en las necesidades concretas de las partes. Un argumento -pero sólo uno- para incrementar la participación de las partes (o los intereses implicados) en el proceso de creación de la norma es darles nuevas oportunidades para articular sus necesidades reales y concretas. De esta manera, también la teoría del Derecho reflexivo puede ser interpretada como partidaria de incrementar la orientación hacia las necesidades en el Derecho ${ }^{8}$.

Las diferentes personas y los diferentes grupos que componen la sociedad tienen necesidades diversas. Un ordenamiento

${ }^{6}$ Teubner, 1983, pág. 275.

${ }^{7}$ Teubner, 1983, págs. 277-278.

${ }^{8}$ El paralelismo (hasta cierto punto) entre las ideas de Eriksson y las de Teubner puede en parte explicarse por el hecho de que en la formulación de sus teorías ambos autores remiten a Jürgen Habermas. Eriksson (1980, pág. 113) afirma que su racionalidad de las necesidades está conectada con la racionalidad comunicativa que, según Habermas, impera en los grupos fuera del sistema de economía de mercado. 
jurídico que tome en consideración las necesidades concretas y reales de los miembros de la sociedad debe, en consecuencia, aceptar, entre otras cosas, la posibilidad de que existan reglas diferentes para personas diferentes (reglas relativas a la persona) ${ }^{9}$. En muchas ramas del Derecho -como en el Derecho social- esta posibilidad resulta evidente. En otras ramas, sin embargo, la persona «abstracta», sin ninguna característica externa al mundo del Derecho, sigue siendo considerada como el sujeto normal en una norma jurídica. En este artículo me propongo, sobre la base de un material jurídico concreto tomado del Derecho finlandés, discutir algunos aspectos del desarrollo del Derecho privado -especialmente del Derecho de responsabilidad civil y del Derecho de contratos- en una dirección en la que las necesidades de las diversas partes son ampliamente reconocidas como relevantes en la $\operatorname{argumentación}^{10}$.

\section{Conceptos orientados hacia la persona en el Derecho privado}

A lo largo del siglo XX la llamada materialización del Derecho ha generado un estilo de regulación que ha conllevado, en mayor medida que antes, diferenciaciones entre personas. En la literatura jurídica de todo el mundo se han discutido muchos ejemplos relacionados con esta tendencia. Entre otros, puede mencionarse al autor germano occidental Manfred Rehbinder, que estudia el desarrollo de la estructura jurídica a partir de la tricotomía estatus-contrato-rol. Mientras que en el Derecho individualista (Derecho formal) el concepto central era el de «persona», basado en la libertad individual, en el Derecho del Estado social se distingue entre personas sobre la base del lugar que ocupan en el sistema social.

En otras palabras, el nuevo Derecho se centra en roles sociales. El hombre no aparece ya en el Derecho como persona abstracta, sino como empresario o trabajador, como trabajador manual o trabajador de cuello blanco, como persona que delinque por primera vez o reincidente. Cuanto mayor sea la diferenciación en

${ }^{9}$ Aunque este artículo versa fundamentalmente sobre las diferencias personales en el Derecho como un aspecto de la orientación hacia las necesidades, hay que recalcar que una discusión de conjunto sobre la racionalidad de las necesidades en el Derecho no puede y no debe limitarse sólo a este aspecto.

${ }^{10}$ El artículo está basado en mi libro Social civilrätt (Derecho civil social) (1987). 
la vida social, se deberán desarrollar un número tanto mayor de roles de este género ${ }^{11}$. En Escandinavia muchos autores han apuntado el aumento de las disciplinas jurídicas relativas a la persona como el Derecho de los niños, el de los extranjeros, el de los presos, el de los enfermos, el de los consumidores y el de las mujeres ${ }^{12}$.

Dado que en el referido debate los ejemplos de diferenciación personal -que varían de un autor a otro- se presentan, a menudo, de manera poco analítica, conviene aquí realizar un análisis preciso y una tipificación de los conceptos especiales.

Como las normas relativas a personas, por lo general, se introducen en el sistema jurídico con el objetivo de proteger a la «parte más débil» de la relación jurídica, el análisis se centrará en los conceptos mediante los que las normas describen a las referidas partes débiles.

a) Conceptos formales. A menudo, la protección de la parte más débil se realiza a través de normas que utilizan conceptos formales relativos a la persona. El contenido de la regulación es expresión de una racionalidad material, pero su metodología es todavía formal. El campo de aplicación de las normas protectoras de la parte más débil se delimita mediante la diferenciación de los diversos tipos de contratos. En un contrato de seguros, el titular de una póliza está protegido sea la parte más débil o no, sea una persona física o una empresa. A pesar de que en la literatura jurídica suele calificarse a tales regulaciones como «orientadas hacia la persona», en realidad sólo aparentan una diferenciación entre personas diferentes. Las personas protegidas son completamente intercambiables.

b) Conceptos relativos a la función. Durante las últimas décadas se han desarrollado conceptos que hacen referencia a una parte en un contrato y a la función que esa parte cumple en el mercado. Dentro de este tipo de conceptos relativos a la función, el más importante es el de consumidor. Este concepto está empezando -al menos en los países nórdicos- a ganarse el estatus de punto de partida central en la sistematización del Derecho de contratos. Pero este concepto todavía no es realmente un concepto relativo a la persona, puesto que toda persona (física) puede actuar como consumidor. No es aún expresión de la creación

${ }^{11}$ Rehbinder, 1968. Véase también, p. ej., Rebe, 1978, pág. 54, quien describe los cambios estructurales en el Derecho privado bajo el rótulo «Vom universalen Menschenbild zur sektorialem Rollenerwartung»».

${ }^{12}$ Véase, p. ej., Stang Dahl, 1985, pág. 29; y Eckhoff, 1985, pág. 84. 
de un Derecho privado orientado hacia las necesidades, en el sentido de estar especialmente dirigido a apoyar a los grupos económica y socialmente más débiles de la sociedad.

c) Conceptos relativos a la persona "real». Hay normas que sólo son aplicables a ciertas clases de personas. Como ejemplos de criterios para operar tales limitaciones pueden mencionarse los siguientes: la pericia, la profesión, la riqueza, la clase social y el sexo.

La relevancia de tales criterios viene dada por razones diferentes. En unos casos se da importancia a ciertas propiedades de una persona porque se considera que tales propiedades le confieren una determinada pericia (o, si se hace referencia a las partes más débiles, falta de pericia). En otros casos, sin embargo, se toman en cuenta ciertas propiedades de una persona porque se considera que éstas reflejan alguna necesidad especial de aquella persona. En otras palabras, puede distinguirse entre conceptos orientados hacia las capacidades y conceptos orientados hacia las necesidades. Haciendo un mal uso de una conocida frase, podría decirse que los primeros están conectados con la primera parte y los segundos con la segunda parte del principio «de cada cual según sus capacidades, a cada cual según sus necesidades»».

Los conceptos orientados hacia las capacidades no son demasiado novedosos en el Derecho privado. Las nociones tradicionales de culpa, bona fides, etc., no pueden aplicarse a casos concretos sin una referencia a las capacidades de la parte cuyo comportamiento se juzga. Sin embargo, el desarrollo del Derecho privado lleva a enfatizar aún más los argumentos orientados hacia las capacidades. Así, por ejemplo, en muchos sistemas jurídicos las reglas que aluden a la inclusión en un contrato de cláusulas estándar o aquellas otras que aluden al deber de una de las partes de informar a la otra en el momento de concluir un contrato, parten de la distinción entre personas expertas e inexpertas. El desarrollo en el Derecho germano occidental del concepto de «Berufsrecht»» ${ }^{13}$ es también consecuencia de la tendencia a la diferenciación a partir de las capacidades en el Derecho privado.

En este artículo, sin embargo, me centraré en los conceptos relativos a la persona que están orientados hacia las necesidades. Cuando se usan estos conceptos no se hace alusión al comportamiento de una de las partes, por ejemplo, en el momento de

${ }^{13}$ Véase, p. ej., Hopt, 1983. 
celebrar un contrato; estos conceptos destacan una necesidad especial de la parte como fundamento para concederle una protección jurídica. Como ejemplos de conceptos orientados hacia las necesidades pueden mencionarse pobreza, renta baja, desempleo, enfermedad, etc. Realmente, ¿pueden utilizarse estos conceptos en la argumentación en Derecho privado?

\section{Dos casos concretos}

El estudio de un material jurídico concreto (legislación y trabajos preparatorios, jurisprudencia y doctrina jurídica) tomado del Derecho finlandés y escandinavo de los años setenta y especialmente de los ochenta, muestra muchos fragmentos de modelos de argumentación relativos a la persona y orientados hacia las necesidades. En estos casos se hace, normalmente, referencia explícita a la situación económica de la parte (o a factores que se considera que afectan a su situación económica, como el desempleo). En estos contextos no suelen tomarse en consideración otras necesidades distintas de las económicas (véase, sin embargo, el ejemplo 2, infra.).

En este artículo, que va dirigido a una audiencia interesada ante todo por la teoría del Derecho, no pueden y no deben tratarse todos los detalles de esta evolución ${ }^{14}$. Empezaré la discusión citando dos casos concretos de Finlandia. Estos casos no son en absoluto únicos; en este contexto podrían mencionarse muchos otros.

Ejemplo 1. La ley finlandesa sobre responsabilidad civil contiene una cláusula que autoriza al tribunal a ajustar el montante de los daños cuando el pago de toda la compensación resulte irrazonablemente onerosa para la persona responsable del pago ${ }^{15}$. Esta disposición pretendía ser algo así como una «válvula de seguridad» que permitiera a los tribunales ajustar los daños cuando una «pequeña» negligencia hubiera causado un daño extraordinariamente grave ${ }^{16}$. Sin embargo, la Corte Suprema la ha usado más como un principio general de equidad y ha operado

${ }^{14}$ Para estos detalles, véase el capítulo 3 del libro mencionado en la nota 10.

${ }^{15}$ Vahingonkorvauslaki (31-5-1974/412), 2:1.2. Puede encontrarse una disposición similar en la Skadestândslag sueca (1972:207), 6:2.

${ }^{16}$ Ello queda claramente expresado en los trabajos preparatorios, véase «Hallituksen esitys Eduskunnale vahingonkorvausta koskevaksi lainsäädännöksi», HE, 187/1973, pág. 13. 
ajustes limitando bastante las cantidades por daños (algunos miles de marcos cuando, por ejemplo, una compañía de seguros ha intentado recuperar tales cantidades de una persona individual con bajos ingresos) ${ }^{17}$. En otras palabras, la práctica del tribunal en relación con la disposición en cuestión ofrece una buena base para la creación de conceptos orientados hacia las necesidades; el «deudor pobre» en alguna medida (muy limitada en la práctica de los tribunales inferiores) está protegido explícitamente (y no «oculto» por medio del uso de técnicas jurídicas convenientes para el caso) por los tribunales en el Derecho de la responsabilidad civil. Ello se da particularmente cuando la parte contraria es rica como, p.e., una compañía de seguros que hace uso de su derecho de subrogación.

De entre los casos mencionados anteriormente la orientación hacia las necesidades es muy clara en la KKO 1979 II 14. En este caso el conductor A se había saltado un semáforo en rojo y había causado daños a otra persona. Los daños fueron pagados por la compañía de seguros de A que, de acuerdo con la ley finlandesa 20 que regula los seguros en el tráfico automovilístico, tenía un derecho de subrogación contra la persona que hubiera causado el daño cuando ésta hubiera actuado con negligencia grave. La compañía de seguros se personó en el procedimiento criminal en el que A fue condenado por negligencia grave en la conducción y exigió de A el pago de las cantidades. La Corte Suprema consideró que en este caso la compañía de seguros tenía derecho de subrogación (lo cual era bastante

${ }^{17}$ La siguiente tabla muestra los casos en los que la Corte Suprema ha ajustado las cantidades por aplicación de la cláusula general antes mencionada durante los años 1979-1985:

Compensación completa/Fmk

Cantidad ajustada,: Fmk

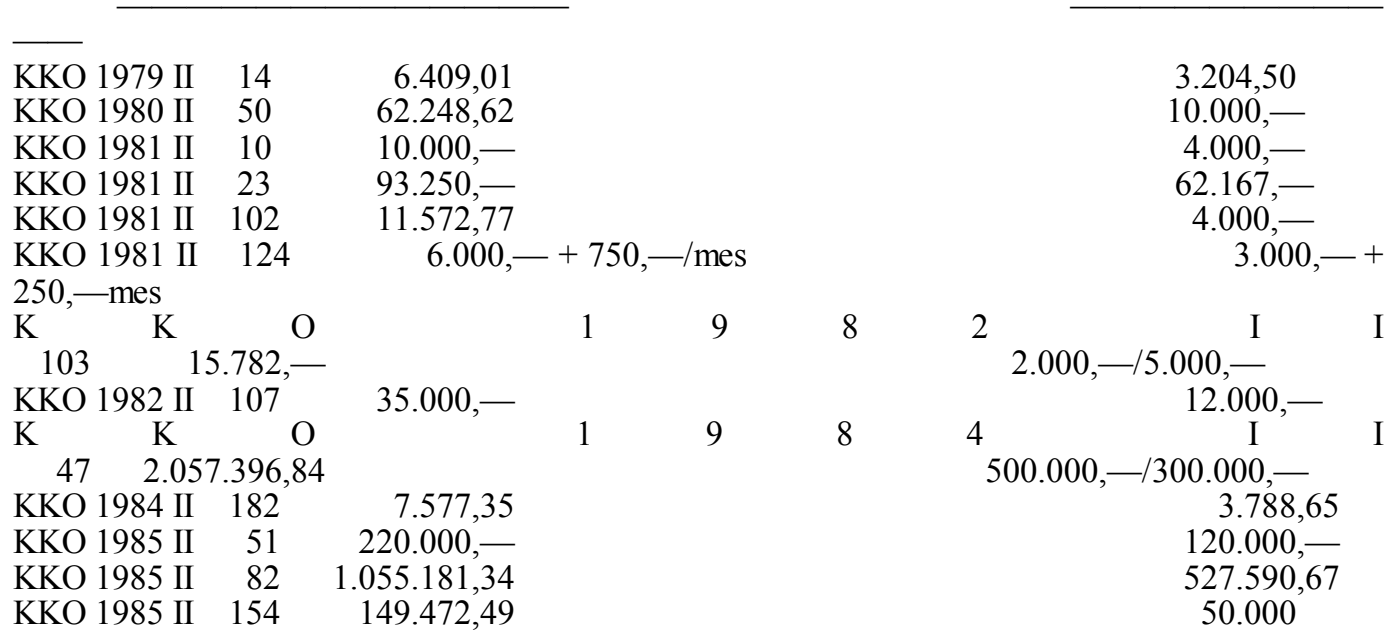


obvio), pero ajustó la cantidad que A debía pagar apoyándose tan sólo en los dos argumentos siguientes: A tenía unos ingresos bajos y un deber de manutención. Lo interesante de este caso radica en que no se podían haber usado como argumentos para este ajuste ni el bajo nivel de culpa (A había incurrido en negligencia grave) ni una cantidad de daños extraordinariamente elevada (la cantidad reclamada era tan sólo de 6.409 Fmk, unos 1.000 dólares). El ajuste, que consistió en pasar de 6.409 Fmk a $3.204 \mathrm{Fmk}$, se fundamentó únicamente en argumentos orientados hacía las necesidades: A tenía unos ingresos bajos (entre 1.800 y 2.800 Fmk-mes) y unos elevados gastos de manutención (tenía cuatro hijos).

Ejemplo 2. La ley finlandesa de contratos contiene una cláusula general según la cual un contrato injusto puede ser ajustado por el tribuna ${ }^{18}$. Cláusulas generales similares a ésta pueden encontrarse en alguna legislación especial como, por ejemplo, la relativa a arrendamientos de pisos. Tales cláusulas generales pueden constituir una vía útil para introducir subrepticiamente argumentos orientados hacia las necesidades también en el Derecho privado.

Nuestro ejemplo proviene de la Corte de Apelación de Helsinki: Hels. Ho 29.6.1977/81. A era propietario de un piso y lo alquiló el 18.9.1976 a B y C. De acuerdo con lo estipulado las cláusulas del contrato debían estar en vigor hasta el 1.1.1980. Durante un tiempo A pasó a vivir como subarrendatario en el interior de otra familia. Algún tiempo después de firmado el contrato, A quiso volver a su piso. La corte de apelación consideró que A, que era viejo (nacido en 1896), no podía adaptarse a vivir como subarrendatario en el interior de una familia con cuatro niños menores de diez anos y se remitió a la declaración de un doctor según la cual vivir en esas condiciones ponía en peligro su salud. Sobre esta base se consideró que el período establecido en el contrato firmado entre A y B-C era injusto contra A y se le concedió el derecho a terminar el contrato antes del 1.1.1980. En este caso una disposición contractual que, en general, era bastante aceptable fue rechazada atendiendo a las necesidades especiales relativas a la persona de A (su avanzada edad y enfermedad).

18 Laki varallisuusoikeudellisista oikeustoimsta, 36 (como enmienda 17-12-1982/956). Disposiciones similares pueden encontrarse en las leyes de contratos de los otros países escandinavos. 


\section{Discusión}

Los ejemplos anteriores -y muchos otros que aquí no se han mencionado- muestran que dentro de la estructura predominante del Derecho privado es posible ya una argumentación relativa a la persona y orientada hacia las necesidades. Por otra parte, es fácil mostrar muchas dificultades conectadas con un posible incremento de esta orientación hacia las necesidades en el Derecho privado. Estas dificultades son frecuentemente destacadas cuando pasamos del campo del Derecho de la responsabilidad civil -del que han sido tomados muchos de nuestros ejemplos- a la rama central del Derecho privado, el llamado Derecho de contratos. Aquí discutiré dos problemas centrales:

a) En el Derecho privado tradicional la relación se da normalmente entre dos partes cuyos intereses, en el caso de que surja una disputa, se oponen el uno al otro. Si una parte gana, la otra pierde. En este campo, la satisfacción de las necesidades de una parte se realiza a expensas de la otra. Si una persona pide una solución orientada hacia las necesidades está, por tanto, obligada a argumentar por qué y en qué situaciones la otra parte debe financiar la satisfacción de sus necesidades. Ello, a su vez, significa que la argumentación orientada hacia las necesidades sólo puede prosperar en ciertos casos: en aquellos en los que la referida carga argumentativa puede ser satisfecha. Cuando una necesidad se opone a una necesidad (igual) no podemos hallar una solución orientada hacia las necesidades.

En el primero de los casos antes citados, la parte contraria era una compañía de seguros (notoriamente más rica); en muchos -aunque no en todos- de los casos de Derecho de responsabilidad civil en que se producían ajustes la situación era la misma. Puede decirse que la relación más típica en la que será posible una solución relativa a la persona y orientada hacia las necesidades en el Derecho privado es la relación entre una persona física y una (gran) empresa (o el Estado o alguna sociedad pública). En la mayoría de los casos concretos, e incluso en otros materiales tomados de otras partes del Derecho privado -p. e., del Derecho de contratos-, en que ha operado una argumentación orientada hacia las necesidades se encuentran relaciones de este tipo ${ }^{19}$.

${ }^{19}$ La tradicional orientación hacia las necesidades en el Derecho de familia (p. ej., en las reglas concernientes a las pensiones de alimentos) no puede ser analizada en este contexto. 
Algunos de los argumentos concernientes a la responsabilidad de las empresas, bien conocidos por la doctrina del Derecho privado, pueden también utilizarse para imponer un «deber de tomar en cuenta las necesidades de la otra parte» del tipo antes mencionados sobre empresas (al menos, en el caso de las grandes) y otras entidades semejantes. Este no es el lugar apropiado para tratar con detalle estos argumentos; en este contexto es suficiente con algunas llamadas de atención sobre ciertos puntos básicos:

- la imagen burocrática y el poder económico de las grandes empresas; debería haber alguna conexión entre poder y responsabilidad;

- pulverización de las necesidades; normalmente, a través del mecanismo del precio, una empresa puede repercutir las pérdidas que hayan surgido sobre un gran numero de clientes (consumidores);

—responsabilidad por problemas ocasionados por las empresas; p. e.: Si una compañía financiera introduce un sistema de tarjetas de crédito que causa problemas a aquellas personas que no son capaces de adaptarse al mismo, entonces la referida compañía debería tener alguna responsabilidad por esos problemas.

b) El problema anteriormente tratado se planteaba en el nivel normativo. El segundo problema plantea mayor dificultad y está relacionado con la eficacia de las regulaciones orientadas hacia las necesidades y, en particular, con sus problemáticos efectos secundarios. El problema que voy a tratar aquí no es agudo en el Derecho de la responsabilidad civil (y ello podría explicar por qué las regulaciones orientadas hacia las necesidades parecen ser bastante más comunes en este campo $)^{20}$. Es, fundamentalmente, en el Derecho de contratos donde uno debe considerar el problema de la relación entre las reglas orientadas hacia las necesidades y el mercado.

La cuestión que hay que responder es la siguiente: ¿cómo puede evitarse que los principios relativos a la persona y orientados hacia las necesidades produzcan efectos negativos sobre

${ }^{20}$ En el material tomado del Derecho finlandés en relación con la orientación hacia las necesidades, los ejemplos de Derecho de la responsabilidad civil constituyen una parte predominante. Véase el libro mencionado en la nota 10, pág. 84-114. 
las posibilidades de contratación de las personas protegidas? ¿Cómo puede evitarse que una regulación que, por ejemplo, protege a un deudor en paro, deteriore aún más las posibilidades de obtener crédito por parte de los parados? Naturalmente, una respuesta puede ser la de tratar de eliminar los efectos negativos del mercado desarrollando reglas que establezcan contratos obligatorios. Sin embargo, en este contexto no voy a discutir esta alternativa.

Los efectos que se acaban de mencionar no se originan cuando las necesidades relativas a la persona que tratan de protegerse son del tipo de las llamadas «necesidades sobrevenidas», esto es, necesidades que surgen con posterioridad a la celebración del contrato. En este caso uno puede acudir a algunas normas jurídicas concretas ${ }^{21}$ que protegen, hasta cierto punto, al deudor contra las sanciones demasiado elevadas cuando no puede pagar la deuda por causas sobrevenidas tras la celebración del contrato, como enfermedad, paro, etc. En estos casos puede hablarse de un desarrollo doctrinal de la fuerza mayor social ${ }^{22}$.

Los efectos negativos de una regulación orientada hacia las necesidades sobre las posibilidades de contratación de los grupos de personas protegidas son probablemente mayores cuando $a$ ) el grupo es relativamente pequeño, $b$ ) es fácil delimitarlo frente a otros grupos, y c) las consecuencias jurídicas de la regulación son claras. Cuando la protección se realiza mediante cláusulas generales, cuya aplicación es hasta cierto punto incierta, el riesgo de los efectos negativos disminuye. La mayoría de los casos orientados hacia las necesidades tomados del Derecho finlandés y escandinavo son de esta clase; son casos decididos sobre la base de cláusulas generales más o menos abiertas en las que la predecibilidad del resultado era extremadamente baja. Los conceptos relativos a la persona y orientados hacia las necesidades desarrollados sobre la base de estos casos son conceptos-graduables, que designan propiedades que pueden darse en mayor o menor grado (p. e, la pobreza), y conceptos-directriz, que deben balancearse con otros argumentos a la hora de determinar las consecuencias jurídicas de su aplicación.

\footnotetext{
${ }^{21}$ Un ejemplo: la ley finlandesa que regula los intereses (Korkolaki, 20-8-1982/633), § 11, confiere a los tribunales el poder de delimitar la responsabilidad en el pago de intereses de demora cuando la causa de la demora sea enfermedad o desempleo del deudor o alguna otra razón semejante.

${ }^{22}$ Desde un punto de partida diferente -una «interpretación social» del Bürgerliches Gesetzbuch-, Udo Reifner (1979, pág. 319) ha recomendado una doctrina similar.
} 
Ello no obstante, esta exigencia económica está en cierto sentido en línea con las ideas básicas implicadas por la orientación hacia las necesidades. Como una solución orientada hacia las necesidades es aquella que toma en cuenta las necesidades reales y concretas de las partes, esa solución, hasta cierto punto, siempre se apoya obviamente en un juicio in casu. Ello sitúa a los tribunales en el centro de nuestro interés.

Es obvio que un adecuado programa orientado hacía las necesidades presupone una regulación flexible. Por otro lado, no hay, sin embargo, garantías de que los tribunales utilicen en la práctica un programa flexible de promoción de los intereses de los grupos sociales con menos recursos. Por tanto, desde el punto de vista de la doctrina crítica, el objetivo debería ser la creación de un modelo de argumentación que, en la medida de lo posible, combinara el contenido orientado hacia las necesidades de algunos nuevos elementos del Derecho privado con el valor formal de la seguridad jurídica.

Esto puede efectuarse mediante el desarrollo de los principios generales y de los conceptos orientados hacia las necesidades, clarificando nuevos puntos capitales de la argumentación jurídica de forma que se fortalezca la seguridad jurídica, pero tratándolos como meras directrices, de forma que puedan reflejar adecuadamente las necesidades concretas de las partes. De esta manera, la doctrina puede suministrar, cuando menos, los instrumentos necesarios para una práctica orientada hacia las necesidades ${ }^{23}$.

(Trad. de Josep Aguiló Regla)

\footnotetext{
${ }^{23}$ Una posibilidad que no puede negarse es que la función primaria de esta teoría sea legitimadora; en palabras de Roberto Mangabeira Unger (1976, pág. 213): «En muchos países, las teorías jurídicas de justicia sustantiva pueden permanecer aisladas en una atmósfera políticamente hostil de forma que, si bien pueden tener la suficiente vitalidad como para contribuir a legitimar el orden social, nunca pueden fortalecerse lo suficiente para ayudar a transformarlo.»
} 


\section{BIBLIOGRAFÍA}

Brúggemeier, Gert, «Wirtschaftsverfassung und Staatsverfassung», «Mischverfassung des demokratischen Interventionskapitalismus», «Verfassungstheorie des Sozialstaates», Drei Modelle der Verflechtung von Staat und Wirtschaft? Eine Problemskizze. Rechtsformen der Verflechtung von Staat und Writschaft, herausgegeben von Volkmar Gessner und Gerd Winter, Opladen, 1982, págs. 60-73.

Eckhoff, Torstein, «Kan vi laere noe av kvinneretten?», Retfaerd 31 (1985), págs. 82-90.

Eriksson, Lars D., «Utkast till en marxistisk jurisprudens», Retfaerd 11 (1979), págs. 40-53.

—, Marxistisk teori och rättsvetenskap, Helsinki, 1980.

Hopt, Klaus, «Nichtvertragliche Haftung ausserhalb von Schadensund Bereicherungsausgleich», Zur Theorie und Dogmatik des Berufsrechts und der Berufshaftung, Archiv für die civilistische Praxis 183 (1983), págs. 608-720.

Mathiesen, Thomas, «“Styringsjuss”, "rettssikkerhetsjuss” ogdet gode samfunn», Retfaerd 28 (1985), págs. 12-25.

Rebe, Bernd, Privatrecht und Wirtschaftsordnung, Bielefeld, 1978.

Rehbinder, Manfred, Status-Kontrakt-Rolle. Berliner Festschrift für Ernst E Hirsch, Berlín(Oeste), 1968, págs. 141-169.

Reifner, Udo, Alternatives Wirtschaftsrecht am Beispiel der Verbraucherverschuldung, Darmstadt, 1979.

Rottleuthner, Hubert, «Theories of legal evolution: between empiricism and philosphy of history», Rechtstheorie Beiheft 9 (1986), págs. 217-230.

Schmidt, Eike, Von der Privat-zur Sozialautonomie, Juristenzeitung, 1980, págs. 153-161.

Stang Dahl, Tove, Innledning til kvinneretten, Stang Dahl (ed.), Kvinnerett I. Oslo, 1985, págs. 13-109.

Teubner, Gunther, «Substantive and Reflexive Elements in Modern Law», Law \& Society Review, vol. 17 (1983), págs. 239-285.

Unger, Roberto Mangabeira, Law in Modern Society, New York, 1976.

Wiethölter, Rudolf, «Entwicklung des Rechtsbegriffs», Rechtsformen der Verflechtung von Staat und Wirtschaft, herausgegeben von Volkmar Gessner und Gerd Winter, Opladen, 1982, págs. 38-59.

—, «Materialisierungenund Prozeduralisierungen von Recht», Zentrum für Europäische Rechtspolitik (Bremen), Mat 4 (1984), págs. 25-64.

Wilhelmsson, Thomas, Social civilrätt, Vammala, 1987. 